\title{
Study on the Evaluation of the Exploitation Efficiency of Creative Scientific Talents Based on DEA
}

\author{
Yinling Song a , Chengjun Wang \\ School of Management, Xi'an University of Architecture and Technology, Xi'an 710055, China \\ a1152828599@qq.com
}

Keywords: Creative scientific talents; Comprehensive efficiency; Data envelopment analysis.

\begin{abstract}
Taking Shaanxi Province Youth Science and technology new star plan as an example, establish the input and output index of exploitation on creative scientific talents, use data envelopment analysis method to evaluate the exploitation efficiency about the 45 youth science and technology new stars on this basis. The result shows that the comprehensive efficiency and pure technical efficiency is higher and the scale efficiency is the key to restrict its exploitation efficiency.
\end{abstract}

\section{Introduction}

The level of the exploitation efficiency of creative scientific talents directly related to the social development of science and technology, in the period of rapid development of our country, it is in urgent need of talents, so, it is particularly important to study the exploitation efficiency of creative scientific talents. In recent years, from the national down to the local governments, both have put a lot of resources in the innovative talents training, implement a series of talents plan. But how about the input and output efficiency of these plans in the end? What influences it? Could from which aspects to improve? These are urgent problems to be solved.

Scholars study the exploitation efficiency of creative scientific talents from different angles, Jiang Yan (2011) take the Taishan scholar construction project as an example, to evaluate the efficiency of inputs and outputs, and proposed changes to promote talents development efficiency based on the results ${ }^{[1]}$. Ding Gang (2012) evaluate the efficiency of inputs and outputs from national wide, pointing out that there are significant differences between the provinces and uneven trend is more prominent ${ }^{[2]}$. Zhang Chunhai (2013) from an environmental perspective, using data envelopment analysis to study the the influence of environmental factors on the exploitation efficiency ${ }^{[3]}$. Firstly, establish the input and output indicators and on this basis, use data envelopment analysis to evaluate the efficiency of the innovative talents.

\section{Model}

DEA model profiled. The DEA model, which has an absolute advantage in the evaluation of the effectiveness of multi input-output, is an operations research method to measure the relative efficiency with the same target "operating unit" or "department", putting forward in twentieth Century at the end of seventy's. The basic idea is to take the object, which is to be evaluated, as a decision making unit DMU, by judging whether the DMU is in the production frontier surface consists of the envelope, or deviating from the production frontier surface to determine the efficiency of DMU [4]. the most commonly used is CCR model with constant returns to scale and BCC model which is add the convexity assumptions on the basis of CCR model.

Model establishment. This paper establishes the innovative talents, exploitation efficiency mode based DEA [5-6]. Suppose there is $m$ innovative talents, the corresponding input index by $X$, the output index by $\mathrm{Y}$, each person corresponds to $n$ inputs and $p$ outputs, the $n$ inputs and $p$ outputs can be expressed in vector respectively as follows:

$$
\begin{aligned}
& X=\left(x_{1}, x_{2} \cdots \cdots x_{n}\right)^{T} \\
& Y=\left(y_{1}, y_{2} \cdots \cdots y_{p}\right)^{T}
\end{aligned}
$$


Among them, $x_{1}$ represents the first input, $x_{2}$ represents the second input, so $x_{n}$ represents the $n$-th input. Similarly, $y_{1}$ represents the first output, $y_{2}$ represents the second output, so, $y_{p}$ represents the $n$ - th output.

Making unit efficiency model based on input is:

$$
\begin{aligned}
& \min \left[\theta-\varepsilon\left(\sum_{j=1}^{n} S_{j}^{-}+\sum_{r=1}^{p} S_{r}^{+}\right]\right. \\
& \sum_{i=1}^{m} \lambda_{i} x_{i j}+S_{j}^{-}=\theta x_{i j} \\
& \sum_{i=1}^{m} \lambda_{i} y_{i r}+S_{r}^{+}=y_{i r} \\
& \sum_{i=1}^{m} \lambda_{i}=1 \\
& \lambda_{i} \geq 0 ; S_{j}^{-} \geq 0 ; S_{r}^{+} \geq 0 ; i=1,2 \cdots \cdots m ; \theta \text { unconstrained }
\end{aligned}
$$

In the above formula, $\theta$ refers to the comprehensive efficiency( $T E), \theta \in(0,1] ; \varepsilon$ represents a non-Archimedean infinitesimal, general take $\varepsilon=10^{-7} ; S_{j}^{-}$and $S_{r}^{+}$refers to the slack variables of input and output, indicating lack of inputs and outputs of redundancy; $\lambda_{i}$ refers to weight; ${ }_{i j}$ refers to the $i$ - th innovative talents the $j$ - th type resources invested; $y_{i r}$ refers to the $i$ - th innovative talents the $i$ type resources invested. This study regulations, $\theta=1$, namely the value of $T E$, represents the efficiency of decision making units; $\theta \neq 1$, invalid. In this invalid decision making units, $\theta \geq 0.9$, represents the exploitation efficiency of the innovative talents is very high; $\theta \geq 0.8$, represents relatively high; $\theta<0.8$, represents the exploitation efficiency is insufficient. The value of the $P T E$ and $S E$ is same with TE .

\section{Empirical study}

To accelerate cultivating of the innovative talents of outstanding young scientists, in 2009, Shaanxi established a special breeding programs-- "Youth Science and Technology Star". In this study, we have design a questionnaire of the new star, including the basic information, funded and work in the training period, the achievements and so on, distribution the questionnaire to all the stars. A total of 320 questionnaires, 291 valid questionnaires, and the effective questionnaire was $90.94 \%$. Considering the training period is three years, and the output will take some time to reflect, so we randomly selected a total of 45 talents in 2011, 2012 and 2013 for the study sample, in the sample, 36 men, and accounting for the total sample 80\%; with Dr and above 42 people, accounting for $93.33 \%$ of the total sample.

Index selection. Through literature review, the output index we chose is academic papers, invention patent, talents cultivation, achievements transformation, awards above provincial.The input index we chose is government special fund, project funds, company invested funds, activities funds, relevant security measures.

DEA model analysis results.(1)Input-output efficiency analysis of the innovative talents. This article selects the DEA model based on investment orientation, use DEAP2.1 software to analyze data, and get the results are shown in table 1:

Selected 45 stars, comprehensive efficiency of the mean value of 0.809 , therefore, the talents overall development efficiency is higher, among them, 17 people, the comprehensive efficiency value is 1 , accounted for $37.78 \%$ of the total. Pure technical efficiency of the mean value of 0.938 , therefore, the talents overall development of pure technical efficiency is very high, among them, 26 people, the pure technical efficiency value is 1 , accounting for $57.78 \%$ of the total. Nine people pure technical 
efficiency value is 1 but scale efficiency value is not 1 . Scale efficiency of mean value of 0.854 , among them, 20 people, and the scale efficiency value is 1 , accounted for $44.44 \%$ of the total. Three people, scale efficiency value is 1 but pure technical efficiency value is not 1 . Among the sample, increasing return of scale have 20 people, account for $44.44 \%$ of the total, scale diminishing returns five people, accounted for $11.11 \%$ of the total, scale constant return 20 people, accounted for $44.44 \%$ of the total.

Table 1 The input and output efficiency results of the innovative talents

\begin{tabular}{c|c|c|c|c|c|c|c|c|c}
\hline DMU & TE & PTE & SE & PTS & DMU & TE & PTE & SE & PTS \\
\hline 1 & 0.807 & 0.807 & 1.000 & - & 22 & 0.493 & 0.857 & 0.575 & irs \\
\hline 2 & 0.700 & 0.820 & 0.854 & irs & 23 & 0.963 & 1.000 & 0.963 & drs \\
\hline $3^{*}$ & 1.000 & 1.000 & 1.000 & - & 24 & 0.817 & 1.000 & 0.817 & irs \\
\hline 5 & 0.542 & 0.960 & 0.564 & irs & 26 & 0.692 & 0.807 & 0.858 & irs \\
\hline 7 & 0.530 & 0.827 & 0.641 & irs & 27 & 0.927 & 1.000 & 0.927 & irs \\
\hline 9 & 0.940 & 1.000 & 0.940 & drs & 30 & 0.606 & 0.892 & 0.680 & irs \\
\hline 10 & 0.538 & 0.814 & 0.661 & irs & 33 & 0.489 & 0.863 & 0.567 & irs \\
\hline 11 & 0.848 & 0.848 & 1.000 & - & 34 & 0.960 & 1.000 & 0.960 & irs \\
\hline 13 & 0.583 & 1.000 & 0.583 & irs & 35 & 0.583 & 0.828 & 0.705 & irs \\
\hline 14 & 0.630 & 0.772 & 0.816 & irs & 37 & 0.523 & 0.923 & 0.567 & irs \\
\hline 15 & 0.940 & 1.000 & 0.940 & drs & 39 & 0.444 & 0.821 & 0.541 & irs \\
\hline 16 & 0.940 & 1.000 & 0.940 & drs & 41 & 0.432 & 0.828 & 0.522 & irs \\
\hline 17 & 0.471 & 0.861 & 0.547 & irs & 42 & 0.872 & 0.872 & 1.000 & - \\
\hline 18 & 0.940 & 1.000 & 0.940 & drs & 45 & 0.523 & 0.825 & 0.635 & irs \\
\hline 21 & 0.681 & 0.985 & 0.692 & irs & & & & & \\
\hline
\end{tabular}

Note: (1) DMU on the table represent the number of talents; (2) $3 *$ including DMU for a total of 17 , respectively is the sample number $3,4,6,8,12,19,20,25,28,29,31,32,36,38,40,43,44$, (3) TE represents the comprehensive technical efficiency, also known as the technical efficiency, PTE represents the pure technical efficiency, SE refers to the scale efficiency, RTS refers to the scale reward, among them, the "irs" represents the scale of diminishing returns, "DRS" represents the scale increasing returns, "-" represents the scale for the same.

(2)Input and output redundancy analysis for the talents

In the DEA model, invalid decision unit is too much or too little resources input or output. In reality, we are more concerned about how much to put or less output in specific. Through the slack variables of input and output, we can be quantified amount of output or redundancy. Table 2 shows the redundancy of input-output analysis of young talents.

Table 2 The redundancy of input-output analysis

\begin{tabular}{c|c|c|c|c|c|c|c|c|c}
\hline \multicolumn{3}{c}{ Input indicators redundancy rate(\%) } & \multicolumn{4}{c}{ Output indicators redundancy rate(\%) } \\
\hline Inp1 & Inp2 & Inp3 & Inp4 & Inp5 & Otp1 & Otp2 & Otp3 & Otp4 & Otp5 \\
\hline 23.560 & 16.315 & 11.957 & 12.538 & 7.341 & 10.426 & 13.224 & 0.798 & 14.891 & 2.064 \\
\hline
\end{tabular}

On the input side. Government special funds and project funding issue redundancy higher, respectively, $23.560 \%$ and $16.315 \%$, indicating its low efficiency. Scientific and technological activities, project funding issue redundancy rate of $12.538 \%$ and $11.957 \%$, are relatively lower compared with the previous indicators, but also at a slightly high lever. Relevant safeguards redundancy is the lowest, just $7.341 \%$, indicating that the relevant safeguards provided by the government has been more fully utilized. In terms of output, the inadequate rate of achievements and patents is higher, respectively $14.891 \%$ and $13.224 \%$, the papers is $10.426 \%$, the inadequate rate of provincial reward and personnel training is $2.064 \%$ and $0.798 \%$. 


\section{Conclusion}

Using DEA model to evaluate the development efficiency of the talents, and has get the following conclusions:

(1) Using DEA model to evaluate the comprehensive technical efficiency, the pure technical efficiency and the scale efficiency. The results show that there is $62.22 \%$ of the sample, which comprehensive technical efficiency is greater than $0.8,37.78 \%$ of the sample is 1 . The development efficiency of the talents is in a high level

(2) The scale efficiency is a major factor restricting the development efficiency of the talents, and the pure technical efficiency is closely to 1,therefore, by enhancing the ability to take full advantage of the resources are no longer evident, we need to make efforts to enhance the scale efficiency.

\section{Acknowledgement}

This study is supported by the Shaanxi Science and Technology nova project named Nova Youth Science and Technology Policy Evaluation and Management System in Shaanxi Province (2013KJXX-97) and the Shannxi province soft science research project named Study on Shanbei of energy chemical industry base supporting capacity for human resource(2010KRM61).

\section{References}

[1] Jiang Yan, Zhang Ti-Qin. High-level innovative talents development efficiency based on DEA Take Shandong province Taishan Scholar construction project as an example[J]. Shandong Social Sciences, 2011,11:131-134.

[2] Ding Gang, Luo Yuan. Provinces into innovative technology personnel construction output efficiency evaluation and its spatial correlation pattern - Based on DEA model and LISA method [J]. Northwest Population,2012,4(33):13-22.

[3]Zhang Chun-Hai. Regional science and technology and its effects on human development efficiency --- An Empirical Analysis of Provincial Panel Data factors[J]. Technology and Economy,2013,6(3):81-85.

[4] Fan Chiang-Ku, Cheng Shu-Wen, Wu Cheng-Ru. Using GRA and DEA to compare efficiency of bancassurance sales with an insurer' s own team[J]. The Journal of Grey System, 2009 (4):395-406.

[5] Ho, Chien-Ta Bruce. Measuring Dot-Com Efficiency Using a Combined DEA and GRA Approach[J]. Journal of the Operational Research Society, 2011(62):776.

[6] Shaanxi Provincial Youth Science and Technology nova management approach [R], Xi'an: Shaanxi Provincial Science and Technology Department, 2009. 